\title{
Eigenfrequency spectrum of the reinforced concrete cantilever beam
}

\author{
Pavel Morozovskiy ${ }^{1,}{ }^{*}$, Ilya Kulish $^{1}$, Nikita Kryzhanovskii ${ }^{1}$, Vladimir Filatov ${ }^{2}$, and Marina \\ Romanovich $^{1}$ \\ ${ }^{1}$ Peter the Great St. Petersburg Polytechnic University, 195251 St. Petersburg, Russia \\ ${ }^{2}$ Moscow State University of Civil Engineering, 26, Yaroslavskoye shosse, Moscow, 129337, Russia
}

\begin{abstract}
For the calculation of building structures under the influence of external dynamic loads, it is necessary to know the eigen frequencies of the structures themselves in order to prevent such a phenomenon as resonance. Steel has distinctive properties from concrete, so its consideration is necessary to determine the eigen frequency of the whole structure. This article presents the calculation of the cantilever beam analytically and in the software package Abaqus for linear and volumetric problems with the interaction of concrete and reinforcement. Due to the reinforcement, the beam eigen frequencies differ from the eigen frequency of the conventional rigidly fixed rod, as evidenced by the results of the calculation.
\end{abstract}

\section{Introduction}

Resonance is the phenomenon of a sharp increase in the amplitude of forced oscillations, which occurs when the frequency of external action approaches some values (resonance frequencies) determined by the properties of the system. Increase amplitudes are only a consequence of resonance, and the reason is coincidence external (exciting) frequency to internal (eigen) frequency oscillatory systems. Using the phenomenon of resonance can highlight and enhance even the most weak periodic oscillations. The phenomena arising from the resonance, called resonant phenomena [1-3].

At vibrations of the system with the eigenfrequency $\theta=\omega$ at any time, the elastic force of the system is balanced by the inertial force. Since they form a mutually balanced group of forces, they are not able to balance the external load. This can be done only by the strength of inelastic resistance. Due to the smallness of inelastic force, displacements are very large [4]. At resonance, the load, which is balanced only by the internal friction forces, must change in time as well as these forces, i.e. with a shift relative to the elastic forces by a quarter of the period. In the process of resonant vibrations displacements, lagging behind the external force, reach a maximum magnitudes in those moments when the force is zero, and vice versa [5]. The amplification factor, i.e. the value showing how many times the movement at the time from the action of the dynamic force exceeds the movement from the static force, is the formula [6]:

\footnotetext{
* Corresponding author: morozovoff85@gmail.com
} 


$$
\beta=\frac{1}{\sqrt{\left(1-\frac{\theta^{2}}{\omega^{2}}\right)^{2}+\gamma^{2} \frac{\theta^{2}}{\omega^{2}}}}
$$

where $\theta$ - action frequency of external load;

$\omega$ - eigen frequency;

$\gamma$ - coefficient of inelastic resistance (accepted depending on the material of construction).

Thus, in the presence of resistance forces, the external load, which changes in time according to the law of sine and cosine, generates steady-state oscillations, which are represented by the sum of sinusoidal and cosine oscillations with amplitudes A' and A'". Summing, these two oscillations in time give a phase shift by an angle $\varphi 0$ with respect to the change of force. Note that the change in deflections and strength occurs in the "antiphase": their extremes coincide, but the directions are opposite. In this case, the amplitude of the dynamic displacement and the coefficient $\mu$ tend to zero. The above shows that in spite of their relative smallness, inelastic forces play an essential role in resonant or close to them oscillations. The system "turn on" the internal friction forces as a reserve, with the help of which it can only balance the external load at $\theta=\omega$. Therefore, in the resonance zone, the resistance forces must be taken into account. At the same time, outside its accounting, they have little effect on results [7].

For building structures resonance is quite dangerous, as can be seen from the graph. Near 1 , the beam of curves has a very sharp slope (sharp), so at the slightest deviation of the parameters, the amplification factor can increase. Resonance zone - near 1 [8].

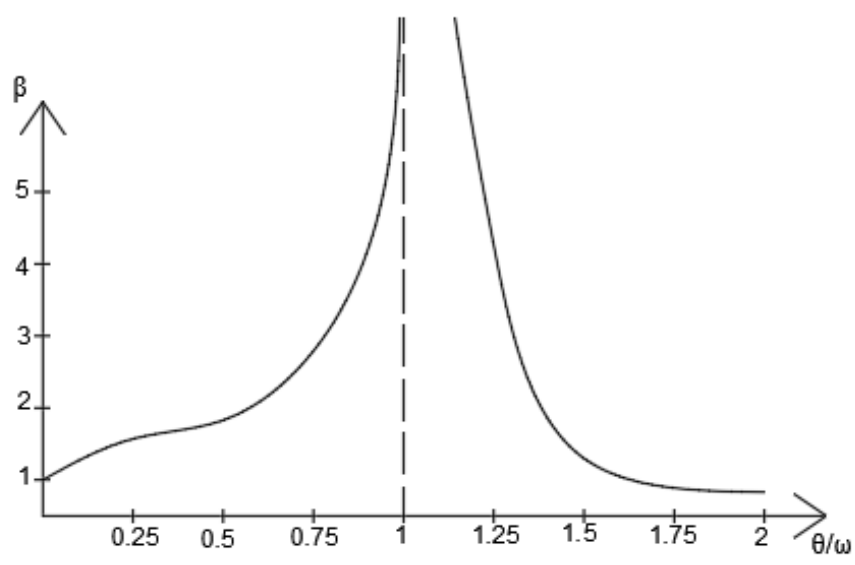

Fig. 1. The amplification factor dependence on the ratio of external and internal frequency.

\section{Calculation}

\subsection{Theoretical calculation}

From the course of the dynamics of structures known formula for determining the eigen frequencies of cantilever beam, depending on the number of the waveform: 


$$
\bar{w}=\frac{3,515^{2}}{l^{2}} \sqrt{\frac{E I * l}{m}}
$$

where 1 - length of beam $(6 \mathrm{~m})$;

$\mathrm{m}$ - mass;

EI- beam stiffness (cross-section is 500x500).

$$
\bar{w}=\frac{3,515^{2}}{36} \sqrt{\frac{27000 * 0.0052 * 6}{37302.4}}=0.0515 \mathrm{~s}^{-1}
$$

We only need the first eigen frequency, since it is the main frequency.

\subsection{Software calculation}

The calculation was made with Simulia Abaqus 2017 software [9].

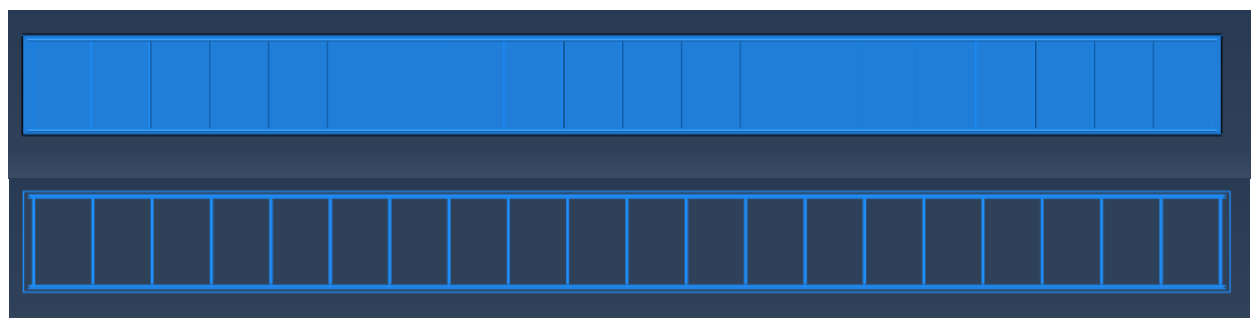

Fig. 2. Model of reinforced beam.

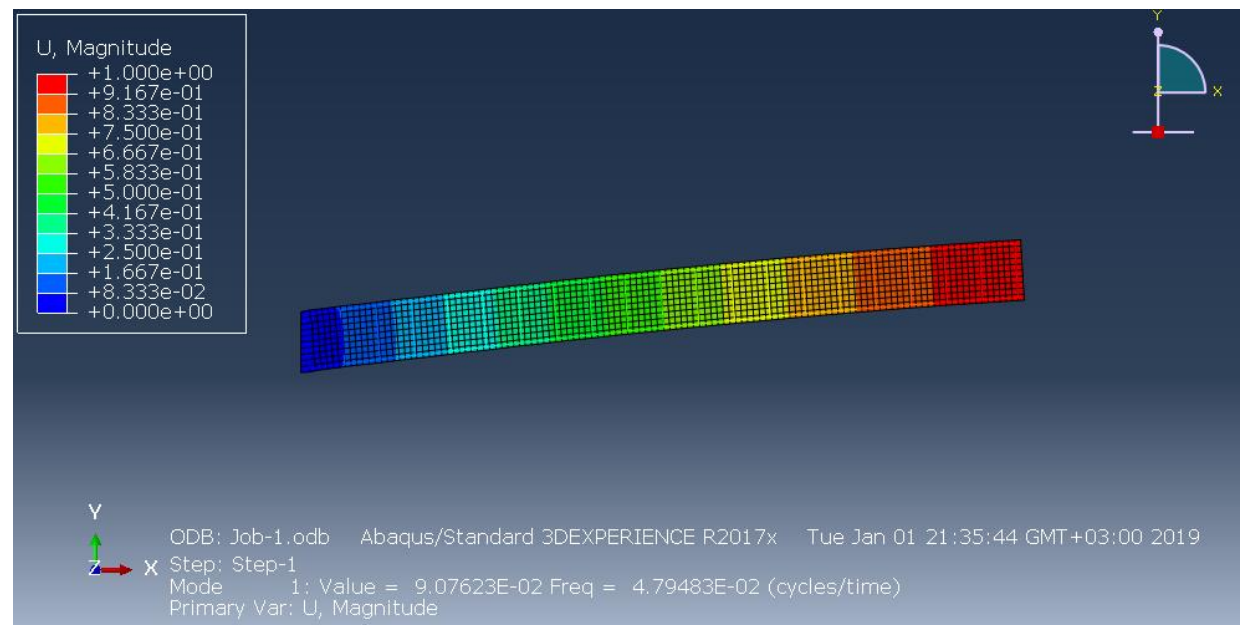

Fig. 3. Calculation result.

\section{Results}

The natural frequency of the two calculations diverges by 7 percent. This result makes it clear that when calculating the dynamic load, it is necessary to take into account the reinforcement of the reinforced concrete beam due to the heterogeneity of the structure itself (Tabl.1) 
Table 1. Eigenfrequency of beam, $\mathrm{s}^{-1}$.

\begin{tabular}{|c|c|c|c|}
\hline $\begin{array}{c}\text { Number of eigen } \\
\text { frequency }\end{array}$ & Analytical & FEM software & $\Delta, \%$ \\
\hline 1 & 0.0515 & 0.0479 & 7.13 \\
\hline
\end{tabular}

The amplification factor's curve is quite curvilinear, so the slightest deviation of the frequency ratio leads to a significant deviation of its factor and the magnitude of the dynamic load as a consequence (Fig.1).

\section{Conclusions}

In this paper, eigen frequencies were found using software and analytical counting for the linear and volume tasks. They have a significant difference, and therefore the results allow us to draw the following conclusion:

It is necessary to take collaboration of steel and concrete to determine the eigen frequency in order to avoid resonance phenomenon, changing the stiffness characteristics of materials and their cross sections.

Also, in order to avoid resonance effects in the building constructions, you can change the design scheme by adding additional hinges, supports, etc.

\section{References}

1. V.A. Kiselev, Structural mechanics: Course. Dynamics and stability of structures. Textbook for universities (Stroyizdat, Moscow, 1980)

2. R. Clough, J. Penzien, Dynamics of buildings (Stroyizdat, Moscow, 1979)

3. I.A. Konstantinov. V.V. Lalin, I.I. Lalina, Dynamics of buildings. Using the SCAD program to solve problems: Studies. Benefit (Publishing house of Polytechnical Institute, $\mathrm{SPb}, 2007)$

4. S.P. Timoshenko, Oscillations in engineering (Science, Moscow, 1967)

5. V.A. Kiselev, Construction mechanics: Special course (dynamics and stability of structures) (Stroyizdat, Moscow, 1980)

6. M.F. Barstein, V.A. Ilyichev, B.G. Koren, Dynamic calculation of buildings and structures (Stroyizdat, Moscow, 1984)

7. A.N. Birbraer, Calculation of structures for earthquake resistance (Science, SPb, 1998)

8. M.A. Birger, J.G. Panovko, Strength, stability, vibrations, Handbook (Mechanical Engineering, Moscow, 1968) 